\title{
ON RADICAL FORMULA IN MODULES
}

\author{
A. NIKSERESHT and A. AZIZI \\ Department of Mathematics, College of Sciences, \\ Shiraz University, Shiraz, 71457-44776, Iran \\ e-mails:a_nikseresht@shirazu.ac.ir,aazizi@shirazu.ac.ir
}

(Received 5 November 2010; revised 1 April 2011; accepted 11 April 2011)

\begin{abstract}
We will state some conditions under which if a quotient of a module $M$ satisfies the radical formula of degree $k$ (s.t.r.f of degree $k$ ), so does $M$. Especially, we will introduce some particular modules $M^{\prime}$ such that $M^{\prime} \oplus M^{\prime \prime}$ s.t.r.f of degree $k$, when $M^{\prime \prime}$ s.t.r.f of degree $k$. Furthermore, we will show that, under certain conditions, if the completion of a module $M$ s.t.r.f of degree $k$, then there is a non-negative integer $k^{\prime}$ such that $M$ s.t.r.f. of degree $k^{\prime}$. Moreover, we state a corrected version of Leung and Man's theorem (K. H. Leung and S. H. Man, On commutative Noetherian rings which satisfy the radical formula, Glasgow Math. J. 39 (1997), 285-293) on Noetherian rings that satisfies the radical formula.
\end{abstract}

2010 Mathematics Subject Classification. 13C99, 13A15, 13 C13.

1. Introduction. In this paper all rings are commutative and with identity, all modules are unitary, $R$ denotes a ring and $M$ denotes an $R$-module. Also, by $\mathbb{N}$ we mean the set of positive integers and $\mathbb{N}^{*}=\mathbb{N} \cup\{0, \infty\}$.

A proper submodule $P$ of $M$ is called prime when from $r m \in P$ for some $r \in R$ and $m \in M$, we can conclude either $m \in P$ or $r M \subseteq P$ (see for example $[\mathbf{1}, \mathbf{3}, \mathbf{4}, \mathbf{6 , 9}, \mathbf{1 0}$, $11,13,15,16])$. Let $(P: M)$ be the set of all $r \in R$ such that $r M \subseteq P$. If $P$ is a prime submodule, then $\mathfrak{P}=(P: M)$ is a prime ideal of $R$ and we say that $P$ is $\mathfrak{P}$-prime. Recall that if $R$ is an integral domain, then $M$ is called torsion-free, if for every $0 \neq r \in R$ and $0 \neq m \in M$, we have $r m \neq 0$. One can easily verify that $P$ is a $\mathfrak{P}$-prime submodule of $M$ if and only if $\frac{M}{P}$ is a torsion-free $\frac{R}{\mathfrak{P}}$-module.

Another generalisation of prime ideals to modules was proposed in [7]. There a proper submodule $W$ of $M$ is said to be weakly prime, if from $r s m \in W$ for $r, s \in R$ and $m \in M$, we can conclude either $r m \in W$ or $s m \in W$. One can easily see that it is equivalent to saying that $(W: m)$ is a prime ideal for every $m \in M \backslash W$. So every prime submodule of $M$ is weakly prime.

Recall that for an ideal $\mathfrak{I}$ of $R$, the intersection of all prime ideals of $R$ containing $\mathfrak{I}$ is called the radical of $\mathfrak{I}$ and is denoted by $\sqrt{\mathfrak{I}}$. Similarly, if $N$ is a submodule of $M$, the intersection of prime (weakly prime) submodules of $M$ containing $N$ is called the radical (weak radical) of $N$ and we denote it by $\operatorname{rad}_{M}(N)$ or $\operatorname{rad}(N)\left(\operatorname{wrad}_{M}(N)\right.$ or $\operatorname{wrad}(N))$.

It is well known that $\sqrt{\mathfrak{I}}=\left\{r \in R \mid r^{k} \in \mathfrak{I}\right.$ for some $\left.\mathrm{k} \in \mathbb{N}\right\}$. In order to find a similar characterisation for $\operatorname{rad}(N)$, the notion of envelope of a submodule was introduced by McCasland and Moore [13]. The envelope of a submodule $N$ of $M$, $\mathrm{E}_{M}(N)$ (or $\mathrm{E}(N)$ if no subtlety), is the set of all $x \in M$ for which there exist $r \in R$, 
$m \in M$ and $k \in \mathbb{N}$ such that $x=r m$ and $r^{k} m \in N$. In general, $\mathrm{E}(B)$ is not a submodule, so we consider $R \mathrm{E}(B)$ (or $R \mathrm{E}_{M}(B)$ ) to be the submodule of $M$ generated by $\mathrm{E}(B)$.

Also in [3], the $k$ th envelope of $N$ was defined recursively by $\mathrm{E}_{0}(N)=N$ and $\mathrm{E}_{k}(N)=\mathrm{E}\left(R \mathrm{E}_{k-1}(N)\right)$, where $R \mathrm{E}_{k}(N)$ (or $\left.R \mathrm{E}_{k, M}(N)\right)$ is a submodule of $M$ generated by $\mathrm{E}_{k}(N)$. Moreover, here by $R \mathrm{E}_{\infty}(N)$ we mean $\bigcup_{i=0}^{\infty} R \mathrm{E}_{k}(N)$.

One can easily verify that for every submodule $N$ of $M$

$$
N=R \mathrm{E}_{0}(N) \subseteq R \mathrm{E}_{1}(N) \subseteq R \mathrm{E}_{2}(N) \cdots \subseteq R \mathrm{E}_{\infty}(N) \subseteq \operatorname{wrad}(N) \subseteq \operatorname{rad}(N) .
$$

Now if $\operatorname{rad}(N)=R \mathrm{E}_{k}(N)\left(\operatorname{wrad}(N)=R \mathrm{E}_{k}(N)\right)$ for a $k \in \mathbb{N}^{*}$, then we say that $N$ satisfies (weakly satisfies) the radical formula of degree $k$, or $N$ s.t.r.f. (weakly s.t.r.f.) of degree $k$ in $M$. A module $M$ s.t.r.f. (weakly s.t.r.f.) of degree $k$, when every submodule of $M$ s.t.r.f. (weakly s.t.r.f.) of degree $k$ in $M$. Also, we say that $R$ s.t.r.f. (weakly s.t.r.f.) of degree $k$, if every $R$-module s.t.r.f. (weakly s.t.r.f.) of degree $k$. For $k=1$ we simply drop $k$ and write $R$ s.t.r.f. (weakly s.t.r.f.) and write $R \mathrm{E}(N)$ instead of $R \mathrm{E}_{1}(N)$.

In [3], it was proved that every arithmetical ring with the finite Krull dimension $k$, s.t.r.f. of degree $k$ and that an arithmetical ring with DCC on prime ideals s.t.r.f. of degree $\infty$. Also, [4, Theorem 2.1] states that every arithmetical ring weakly s.t.r.f. of degree $\infty$. In [16] the rings that s.t.r.f. of degree 0 were named absolutely radical, and it was proved that a ring is absolutely radical if and only if it is the von Neumann regular ring. Moreover, in [4] it is proved that $R$ s.t.r.f. of degree 0 if and only if it weakly s.t.r.f. of degree 0. Also in [9], the characterisation of Noetherian rings that s.t.r.f. is given, which, in Section 2, we will show that it needs a small correction.

A proper submodule $N$ of $M$ is called semi-prime if $\mathrm{E}(N)=N$. It is said that $R$ has the s.p.a.r. property when every semi-prime submodule $N$ of an $R$-module is a radical submodule (that is, $N$ equals an intersection of prime submodules). One can easily see that a proper submodule $B$ of $M$ is semi-prime if and only if $B$ is of the form $R \mathrm{E}_{\infty}(N)$ for some submodule $N$ of $M$. Thus, having the s.p.a.r. property is equivalent to s.t.r.f. of degree $\infty$. In [10], Noetherian rings with the s.p.a.r. property were characterized.

It is known that if $M$ s.t.r.f. (weakly s.t.r.f.) of degree $k$ and $N$ is a submodule of $M$, then $\frac{M}{N}$ s.t.r.f. (weakly s.t.r.f.) of degree $k$. In Section 3 we state conditions on $M$ and $N$ under which the converse of this is true. Especially in Sections (3.4), (3.7), (3.11), (3.14) and (3.16), we will introduce some particular modules $M^{\prime}$ such that $M^{\prime} \oplus M^{\prime \prime}$ s.t.r.f. of degree $k$, when $M^{\prime \prime}$ s.t.r.f. of degree $k$.

Also, we will prove that if $s \in \mathbb{N}$ and $\mathfrak{I}$ is a finitely generated ideal of $R$ such that $\mathfrak{I} \subseteq \sqrt{\left(R \mathrm{E}_{s}(0): M\right)}$ and the $\mathfrak{I}$-adic completion of $M$ s.t.r.f. of degree $t$, then $M$ s.t.r.f. of degree $s+t$ and moreover as an application of this, in (3.21) we will show that for Noetherian rings, having the s.p.a.r. property is equivalent to s.t.r.f. of degree 2. This result generalises Theorem 3.5 and Corollary 2.6 of [4], which state that a Noetherian domain $R$ s.t.r.f. of degree $k$ for some $k \in \mathbb{N}^{*}$, if and only if $R$ is a Dedekind domain.

2. A corrected version of Leung and Man's theorem. In [9] the following theorem is claimed to be proved.

TheOREM 2.1. Let $R$ be a Noetherian ring and $\mathfrak{P}_{1}, \mathfrak{P}_{2}, \ldots, \mathfrak{P}_{n}$ be all the minimal prime ideals of $R$. $R$ s.t.r.f. if and only if $R$ is Artinian or all of the following conditions are satisfied:

(a) $R$ is one dimensional and for each $i=1, \ldots, n, \frac{R}{\mathfrak{P}_{i}}$ is a Dedekind domain and $\mathfrak{P}_{i}$ is the only $\mathfrak{P}_{i}$-primary ideal. 
(b) For $k=1, \ldots, n-1,\left(\bigcap_{i=1}^{k} \mathfrak{P}_{i}\right)+\mathfrak{P}_{k+1}=\bigcap_{i=1}^{k}\left(\mathfrak{P}_{i}+\mathfrak{P}_{k+1}\right)$ if $n \geq 2$.

(c) For all $1 \leq i<j \leq n, R=\mathfrak{P}_{i}+\mathfrak{P}_{j}$ or $\frac{R}{\mathfrak{P}_{i}+\mathfrak{P}_{j}}$ is semi-simple Artinian, if $n \geq 2$.

Now we give a counter example of this theorem. Recall that a ring is called a special principal ideal ring $(S P I R)$ if it has a unique prime ideal which is principal. These rings are sometimes called special primary rings (see [8]).

EXAMPLE 2.2. Let $R_{1}$ be an SPIR, which is not a field, and $R_{2}$ be discrete valuation domain, which is not filed. Set $R=R_{1} \times R_{2}$. By [8, Theorem 9.10] any finite direct sum of SPIR and Dedekind's domains is a ZPI-ring; therefore, $R$ is a ZPI-ring, hence s.t.r.f. by [16, Theorem 2.10]. Also, $R$ is clearly Noetherian and one dimensional.

Now if $\mathfrak{P}_{1}$ is the maximal ideal of $R_{1}$, it is easy to see that $\mathfrak{P}=\mathfrak{P}_{1} \times R_{2}$ is both a maximal and a minimal ideal of $R$. Also, $0 \times R_{2}$ is a $\mathfrak{P}$-primary ideal of $R$ not equal to $\mathfrak{P} \times R_{2}$, which contradicts condition (a) of (2.1).

THEOREM 2.3. For correcting Theorem 2.1, we need to replace condition (a) of this theorem with the following condition:

$\left(a^{\prime}\right) R$ is one dimensional and for each $i=1, \ldots, n$; if $\mathfrak{P}_{i}$ is not maximal, then $\frac{R}{\mathfrak{P}_{i}}$ is a Dedekind domain and $\mathfrak{P}_{i}$ is the only $\mathfrak{P}_{i}$-primary ideal.

Proof. First note that if $R$ satisfies the conditions of (2.1), then by the argument stated in [9], $R$ s.t.r.f. Now let $R$ be a ring that satisfies the conditions of the corrected version of the theorem. If $\mathfrak{M}$ is a maximal ideal of $R$, then $R_{\mathfrak{M}}$ is either Artinian or a one-dimensional local ring that satisfies the conditions of (2.1). Thus, in both cases $R_{\mathfrak{M}}$ s.t.r.f., whence by [9, Theorem 3.4], $R$ s.t.r.f.

Now, suppose that $R$ is a one-dimensional Noetherian ring that s.t.r.f. By a careful reading of proof of $[9$, Corollary 2.3], we see that for every minimal prime ideal $\mathfrak{P}$ of $R$, which is not maximal, $\frac{R}{\mathfrak{P}}$ is the Dedekind domain and $\mathfrak{P}$ is the only $\mathfrak{P}$-primary ideal of $R$ (in fact, in that proof its authors have assumed that if $\mathfrak{P}$ is a minimal prime ideal of a one-dimensional Noetherian ring, then it is not maximal, which is not generally true). So condition ( $\left.\mathrm{a}^{\prime}\right)$ holds.

Now note that if (b) (or (c)) holds locally, then it holds globally. Now if $\mathfrak{P}$ is a height zero prime ideal of $R$, then (b) and (c) hold trivially for $R_{\mathfrak{P}}$. If $\mathfrak{P}$ is a height one prime ideal of $R$, then $R_{\mathfrak{P}}$ is a Noetherian local ring of dimension one and hence by [9, Theorem 4.2], $R_{\mathfrak{P}}$ satisfies (b) and (c). Thus, $R$ satisfies these conditions.

COROLLARY 2.4. If $R$ is a reduced Noetherian ring, then $R$ s.t.r.f. if and only if $R$ has the s.p.a.r property.

Proof. In fact this was noted in [10, p. 33], but there [9, Theorem 1.1] was used, which we showed is incorrect. Using (2.3) instead, one can prove the claim with a similar argument.

3. Quotient of modules and the radical formula. The proof of the following lemma is easy and it is left to the reader.

Lemma 3.1. Let $k \in \mathbb{N}^{*}$ and $N$ be a submodule of $M$. For every submodule L of $M$ containing $N$, we have

$\frac{R E_{k, M}(L)}{N}=R E_{k, \frac{M}{N}}\left(\frac{L}{N}\right), \frac{\operatorname{rad}_{M}(L)}{N}=\operatorname{rad}_{\frac{M}{N}}\left(\frac{L}{N}\right)$ and $\frac{\operatorname{wrad}_{M}(L)}{N}=\operatorname{wrad}_{\frac{M}{N}}\left(\frac{L}{N}\right)$. 
By the above lemma if $M^{\prime}$ is a submodule of $M$ and $M$ s.t.r.f. (weakly s.t.r.f.) of degree $k$, then $\frac{M}{M^{\prime}}$ s.t.r.f. (weakly s.t.r.f.) of degree $k$ too. Here we study some conditions on $M$ and $M^{\prime}$, under which the converse of this statement is true. Throughout this section, we assume that $M^{\prime}$ is a submodule of $M$. Consider the following properties of $M^{\prime}$, where $k \in \mathbb{N}^{*}$ (note that each of $\left(\mathrm{I}_{k}\right)$ and $\left(\mathrm{II}_{k}\right)$ have two versions: one for radicals and another for weak radicals):

$\left(\mathrm{I}_{k}\right)$ For every submodule $N$ of $M, R \mathrm{E}_{k, M}\left(N+M^{\prime}\right)=R \mathrm{E}_{k, M}(N)+M^{\prime}$ and $M^{\prime} \cap$ $\operatorname{rad}_{M}(N) \subseteq R \mathrm{E}_{k, M}(N)\left[M^{\prime} \cap \operatorname{wrad}_{M}(N) \subseteq R \mathrm{E}_{k, M}(N)\right]$

$\left(\mathrm{II}_{k}\right)$ For every submodule $N$ of $M, R \mathrm{E}_{k, M}\left(N+M^{\prime}\right) \cap \operatorname{rad}_{M}(N)=R \mathrm{E}_{k, M}(N)$ $\left[R \mathrm{E}_{k, M}\left(N+M^{\prime}\right) \cap \operatorname{wrad}_{M}(N)=R \mathrm{E}_{k, M}(N)\right]$.

THEOREM 3.2. The following statements are equivalent for all $k \in \mathbb{N}^{*}$ :

(a) $M$ s.t.r.f. (weakly s.t.r.f.) of degree $k$.

(b) There is a submodule $M^{\prime}$ of $M$ such that $\left(I_{k}\right)$ for radicals (weak radicals) holds for $M^{\prime}$ and $\frac{M}{M^{\prime}}$ s.t.r.f. (weakly s.t.r.f.) of degree $k$.

(c) Every proper submodule $M^{\prime}$ of $M$ satisfies $\left(I_{k}\right)$ for radicals (weak radicals).

Proof. We prove the radical version of the statement, the proof of the statement on weak radicals is similar.

(a) $\Rightarrow$ (b), (c): Suppose that $M$ s.t.r.f. of degree $k$ and $M^{\prime}$ and $N$ are arbitrary submodules of $M$. Now $R \mathrm{E}_{k}\left(N+M^{\prime}\right) \cap \operatorname{rad}_{M}(N)=\operatorname{rad}_{M}\left(N+M^{\prime}\right) \cap \operatorname{rad}_{M}(N)=$ $\operatorname{rad}_{M}(N)=R \mathrm{E}_{k}(N)$. Thus, every submodule of $M$ has the property ( $\mathrm{II}_{k}$ ) for radicals. Also by (3.1), every quotient module of $M$ s.t.r.f. of degree $k$.

(b) $\Rightarrow$ (a): Since $\frac{M}{M^{\prime}}$ s.t.r.f. of degree $k$ by (3.1), $R \mathrm{E}_{k}\left(N+M^{\prime}\right)=\operatorname{rad}_{M}(N+$ $\left.M^{\prime}\right)$. Hence, $\operatorname{rad}_{M}(N)=\operatorname{rad}_{M}\left(N+M^{\prime}\right) \cap \operatorname{rad}_{M}(N)=R \mathrm{E}_{k}\left(N+M^{\prime}\right) \cap \operatorname{rad}_{M}(N)=$ $R \mathrm{E}_{k}(N)$.

(c) $\Rightarrow$ (a): Let $N$ be an arbitrary submodule of $M$. First, suppose that $\operatorname{rad}_{M}(N) \neq$ $M$. This means that there exists a prime submodule $P$ of $M$ containing $N$. $P$ being proper, $P$ satisfies $\left(\mathrm{II}_{k}\right)$ for radicals. Thus, $\operatorname{rad}_{M}(N)=P \cap \operatorname{rad}_{M}(N)=R \mathrm{E}_{k}(P) \cap$ $\operatorname{rad}_{M}(N)=R \mathrm{E}_{k}(P+N) \cap \operatorname{rad}_{M}(N)=R \mathrm{E}_{k}(N)$. Now suppose that $\operatorname{rad}_{M}(N)=M$. If $R \mathrm{E}_{k}(N) \neq M$, then let $m \in M \backslash R \mathrm{E}_{k}(N)$ and so $R m \neq M$ as $\operatorname{rad}_{M}(N)=M$. Now $m \in$ $R \mathrm{E}_{k}(N+R m)=R \mathrm{E}_{k}(N+R m) \cap M=R \mathrm{E}_{k}(N+R m) \cap \operatorname{rad}_{M}(N)=R \mathrm{E}_{k}(N)$, which is a contradiction.

LEMMA 3.3. Let $k \in \mathbb{N}^{*}$. Then

(a) The condition $\left(I_{k}\right)\left(\left(I I_{k}\right)\right)$ for radicals implies $\left(I_{k}\right)\left(\left(I I_{k}\right)\right)$ for weak radicals.

(b) The condition $\left(I_{k}\right)$ for radicals (weak radicals) implies $\left(I_{k}\right)$ for radicals (weak radicals).

(c) If ( $\left.I_{1}\right)$ holds for radicals (weak radicals), then for all $0 \neq k \in \mathbb{N}^{*},\left(I_{k}\right)$ holds for radicals (weak radicals).

Proof. (a) Obvious.

(b) If $\left(\mathrm{I}_{k}\right)$ holds for radicals and $N$ is an arbitrary submodule of $M$, then using the modularity law, we get

$$
\begin{aligned}
R \mathrm{E}_{k}\left(N+M^{\prime}\right) \cap \operatorname{rad}(N) & =\left(R \mathrm{E}_{k}(N)+M^{\prime}\right) \cap \operatorname{rad}(N) \\
& =R \mathrm{E}_{k}(N)+\left(M^{\prime} \cap \operatorname{rad}(N)\right)=R \mathrm{E}_{k}(N) .
\end{aligned}
$$

(c) This follows from a simple induction, definition of $R \mathrm{E}_{\infty}(N)$ and the fact that $R \mathrm{E}_{i}(N) \subseteq R \mathrm{E}_{j}(N)$ whenever $i \leq j$ and $N$ is a submodule of $M$. 
Let $R$ be an integral domain. An $R$-module $M$ is called divisible if $r M=M$ for every $0 \neq r \in R$. In [15] a module $M$ was called generalized torsion divisible when $M=\sum_{i \in I} M_{i}$ for submodules $M_{i}$ such that for each $i \in I$ there exists a prime ideal $\mathfrak{P}_{i}$ of $R$ such that $\mathfrak{P}_{i} M_{i}=0$ and $M_{i}$ is a torsion divisible $\frac{R}{\mathfrak{P}_{i}}$-module. The following generalises [15, Theorem 2.5].

COROLlary 3.4. Let $0 \neq k \in \mathbb{N}^{*}$. Suppose that $M^{\prime}$ is a generalised torsion divisible submodule of $M$. If $\frac{M}{M^{\prime}}$ s.t.r.f. (weakly s.t.r.f.) of degree $k$, then $M$ s.t.r.f. (weakly s.t.r.f.) of degree k. Particularly if $M^{\prime \prime}$ is a module that s.t.r.f. (weakly s.t.r.f.) of degree $k$, then $M^{\prime} \oplus M^{\prime \prime}$ s.t.r.f. (weakly s.t.r.f.) of degree $k$.

Proof. Let $N$ be an arbitrary submodule of $M$. By [15, Lemma 2.4], $\mathrm{E}(N+$ $\left.M^{\prime}\right) \subseteq R \mathrm{E}(N)$. Hence, $R \mathrm{E}(N)+M^{\prime} \subseteq R \mathrm{E}\left(N+M^{\prime}\right) \subseteq R \mathrm{E}(N) \subseteq R \mathrm{E}(N)+M^{\prime}$, that is $R \mathrm{E}\left(N+M^{\prime}\right)=R \mathrm{E}(N)$. Also, $M^{\prime} \cap \operatorname{rad}_{M}(N) \subseteq M^{\prime} \subseteq R \mathrm{E}\left(N+M^{\prime}\right)=R \mathrm{E}(N)$. Thus, $M^{\prime}$ satisfies $\left(\mathrm{I}_{1}\right)$ for radicals and the result follows from (3.3) and (3.2).

For every prime ideal $\mathfrak{P}$ of $R$ and every submodule $N$ of $M$, let

$$
\mathcal{F}_{\mathfrak{P}}(N)=\{m \in M \mid s m \in N \text { for some } s \in R \backslash \mathfrak{P}\} .
$$

Evidently $\mathcal{F}_{\mathfrak{P}}(N)=N_{\mathfrak{P}} \cap M$, where $N_{\mathfrak{P}}$ is the localisation of $N$ at $\mathfrak{P}$.

The proof of the following lemma follows from [11, Proposition 2.5].

Lemma 3.5. For every submodule $N$ of $M$, we have $\operatorname{rad}_{M}(N)=\bigcap \mathcal{F}_{\mathfrak{P}}(N+\mathfrak{P} M)$, where $\mathfrak{P}$ runs through all the prime ideals of $R$.

A secondary module $M$ is a non-zero module in which for every $r \in R$, either $r M=M$ or $r^{n} M=0$ for some $n \in \mathbb{N}$. If $M$ is secondary, then $\mathfrak{P}=\sqrt{(0: M)}$ is a prime ideal of $R$ and we say that $M$ is $\mathfrak{P}$-secondary.

A minimal secondary representation of $M$ is an equation of the form $M=\sum_{i=1}^{n} M_{i}$, where each $M_{i}$ is $\mathfrak{P}_{i}$-secondary for distinct $\mathfrak{P}_{i}$ s and none of the $M_{i} \mathrm{~s}$ in the sum is redundant. If $M$ has such a representation, then $M$ is called representable. By [12, Theorem 6.9], the set $\left\{\mathfrak{P}_{i} \mid 1 \leq i \leq n\right\}$ is independent of the specific representation and it is denoted by $\operatorname{Att}(M)$.

Lemma 3.6. Let $M^{\prime}$ be a $\mathfrak{P}$-secondary submodule of $M$. Then $\mathfrak{P} M^{\prime}+\left(M^{\prime}\right.$ : $\mathfrak{P} M) \mathfrak{P} M \subseteq R E(0)$.

Proof. We prove that the generators of the left-hand side are in $R E(0)$. Let $p \in \mathfrak{P}$, $m \in M, m^{\prime} \in M^{\prime}$ and $r \in\left(M^{\prime}: \mathfrak{P} M\right)$. Then $p^{k} M^{\prime}=0$ for some $k \in \mathbb{N}$. Thus, $p m^{\prime} \in$ $R \mathrm{E}(0)$. Also, since $r p m \in M^{\prime}, p^{k+1}(r m) \in p^{k} M^{\prime}=0$ and hence $p(r m) \in R \mathrm{E}(0)$.

THEOREM 3.7. Suppose that $M^{\prime}$ is a $\mathfrak{P}$-secondary submodule of $M, 0 \neq k \in \mathbb{N}^{*}$ and $\frac{M}{M^{\prime}}$ s.t.r.f. (weakly s.t.r.f.) of degree $k$. Then $M$ s.t.r.f. (weakly s.t.r.f.) of degree $k$ if one of the following holds:

(a) $k \geq 2$ and $\left(M^{\prime}: \mathfrak{P} M\right) \nsubseteq \mathfrak{P}$.

(b) $k=1$ and $\left(\mathfrak{P} M^{\prime}: \mathfrak{P} M\right) \nsubseteq \mathfrak{P}$.

Proof. By (3.3) and (3.2), in each case it suffices to show that $M^{\prime}$ satisfies $\left(\mathrm{I}_{k}\right)$ for radicals. So let $N$ be an arbitrary submodule of $M$. First we show that in both cases $R \mathrm{E}_{k}\left(N+M^{\prime}\right)=R \mathrm{E}_{k}(N)+M^{\prime}$. We just need to prove this for $k=1$, and for $k>1$ it is proved inductively. 
Let $x \in \mathrm{E}\left(N+M^{\prime}\right)$. Then $x=r x^{\prime}$, where $r \in R$ and $x^{\prime} \in M$ and there exist $t \in$ $\mathbb{N}, n \in N$ and $m \in M^{\prime}$ such that $r^{t} x^{\prime}=n+m$. We will find an $m^{\prime} \in M^{\prime}$ such that $r\left(x^{\prime}-m^{\prime}\right) \in \mathrm{E}(N)$. If $r \notin \mathfrak{P}$, by $M^{\prime}$ being $\mathfrak{P}$-secondary, there is an $m^{\prime} \in M^{\prime}$ such that $r^{t} m^{\prime}=m$. Thus, $r^{t}\left(x^{\prime}-m^{\prime}\right)=n \in N$ and this $m^{\prime}$ has the required property. If $r \in \mathfrak{P}$, then there is a $t^{\prime} \in \mathbb{N}$ such that $r^{t^{\prime}} M=0$. In this case we choose $m^{\prime}=0$, therefore in this case $r^{t+t^{\prime}}\left(x^{\prime}-m^{\prime}\right)=r^{t^{\prime}} n \in N$ and $r\left(x^{\prime}-m^{\prime}\right) \in \mathrm{E}(N)$. Whence, in both cases $x=r\left(x^{\prime}-m^{\prime}\right)+r m^{\prime} \in R \mathrm{E}(N)+M^{\prime}$. Therefore, every generator of $R \mathrm{E}\left(N+M^{\prime}\right)$ is in $R \mathrm{E}(N)+M^{\prime}$ and thus $R \mathrm{E}\left(N+M^{\prime}\right) \subseteq R \mathrm{E}(N)+M^{\prime}$. The converse inclusion is obvious.

Now in each of the above cases we must prove that $M^{\prime} \cap \operatorname{rad}(N) \subseteq R \mathrm{E}_{k}(N)$.

Let $x_{1} \in M^{\prime} \cap \operatorname{rad}_{M}(N)$. By (3.5), $x_{1} \in \mathcal{F}_{\mathfrak{P}}(N+\mathfrak{P} M)$ and hence there exist $r_{1} \in$ $R \backslash \mathfrak{P}, n \in N$ and $m \in \mathfrak{P} M$ such that $r_{1} x_{1}=n+m$.

(a) By the assumption of this case of the theorem, there exists an $r^{\prime} \in\left(M^{\prime}: \mathfrak{M} M\right) \backslash$ $\mathfrak{P}$. If we set $r^{\prime \prime}=r_{1} r^{\prime}$, then $r^{\prime \prime} \in R \backslash \mathfrak{P}$ and $r^{\prime \prime} x_{1}=r^{\prime} n+r^{\prime} m \in N+\left(M^{\prime}: \mathfrak{P} M\right) \mathfrak{P} M$. Thus, by (3.6) $r^{\prime \prime} x_{1} \in R \mathrm{E}(N)$. Now, because $r^{\prime \prime} \notin \mathfrak{P}$ and $x_{1} \in M^{\prime}$, there is a $y \in M^{\prime}$ such that $r^{\prime \prime} y=x_{1}$. Therefore, $r^{\prime \prime 2} y=r^{\prime \prime} x_{1} \in R \mathrm{E}(N)$ and so $x_{1}=r^{\prime \prime} y \in R \mathrm{E}_{2}(N) \subseteq R \mathrm{E}_{k}(N)$. This means $M^{\prime} \cap \operatorname{rad}(N) \subseteq R \mathrm{E}_{k}(N)$ and hence $\left(\mathrm{I}_{k}\right)$ for radicals holds for $M^{\prime}$, which completes the proof of this case.

(b) By our assumption, there exists an $r^{\prime} \in\left(\mathfrak{P} M^{\prime}: \mathfrak{P} M\right) \backslash \mathfrak{P}$. If we set $r^{\prime \prime}=r^{\prime} r_{1}$, then $r^{\prime \prime} x_{1}=r^{\prime} n+r^{\prime} m$ and $r^{\prime} m \in \mathfrak{P} M^{\prime}$. Put $m^{\prime}=r^{\prime} m$. Then $m^{\prime}=\sum_{i=1}^{t} p_{i} m_{i}^{\prime}$, where $p_{i} \in \mathfrak{P}$ and $m_{i}^{\prime} \in M^{\prime}$. As $r^{\prime \prime} \notin \mathfrak{P}$, for each $i \leq i \leq t$, there exists $m_{i}^{\prime \prime} \in M^{\prime}$ such that $m_{i}^{\prime}=r^{\prime \prime} m_{i}^{\prime \prime}$. Let $m^{\prime \prime}=\sum_{i=1}^{t} p_{i} m_{i}^{\prime \prime} \in \mathfrak{P} M^{\prime}$, which is in $R \mathrm{E}(N)$ by (3.6). Now $r^{\prime \prime} m^{\prime \prime}=m^{\prime}$ and whence $r^{\prime \prime}\left(x_{1}-m^{\prime \prime}\right)=r^{\prime \prime} x_{1}-m^{\prime}=r^{\prime} n \in N$. Moreover, $x_{1}-m^{\prime \prime} \in M^{\prime}$, thus $x_{1}-m^{\prime \prime}=r^{\prime \prime} y$ for some $y \in M^{\prime}$, as $r^{\prime \prime} \notin \mathfrak{P}$. Therefore, $r^{\prime \prime 2} y=r^{\prime \prime}\left(x_{1}-m^{\prime \prime}\right) \in N$ and so $x_{1}-m^{\prime \prime}=r^{\prime \prime} y \in$ $R \mathrm{E}(N)$, and as $m^{\prime \prime} \in R \mathrm{E}(N)$, we have $x_{1} \in R \mathrm{E}(N)$. This means $M^{\prime} \cap \operatorname{rad}(N) \subseteq R \mathrm{E}(N)$, which completes the proof.

Note that if $R$ is an integral domain, then every divisible $R$-module is 0 -secondary. Thus, (3.7) generalises [15, Theorem 2.2], which states if $M$ is a direct sum of a divisible module and a module which s.t.r.f., then $M$ s.t.r.f. Now we will state another application of (3.7).

Proposition 3.8. Let $n \in \mathbb{N}$, and suppose for each $1 \leq i \leq n, M_{i}$ is a $\mathfrak{P}_{i}$ secondary $R$-module such that $M_{i}=M_{j}$ if $\mathfrak{P}_{i}=\mathfrak{P}_{j}$. If $\mathfrak{I}$ is an ideal of $R$ with $\mathfrak{I} \nsubseteq \cup_{i=1}^{n} \mathfrak{P}_{i}$, then $M=M_{1} \oplus M_{2} \oplus \cdots \oplus M_{n} \oplus \frac{R}{\mathfrak{J}}$ s.t.r.f.

Proof. We use induction on $n$. As an application of the previous theorem, we have the result for $n=1$. Now suppose the result is correct for $n-1$.

By rearranging the indices, we may suppose that $\mathfrak{P}_{1}$ is a minimal element of the set $\left\{\mathfrak{P}_{1}, \mathfrak{P}_{2}, \cdots, \mathfrak{P}_{n}\right\}$. Then for each $1 \leq i \leq n$, either $\mathfrak{P}_{i}=\mathfrak{P}_{1}$ or $\mathfrak{P}_{i} \nsubseteq \mathfrak{P}_{1}$. So by our assumption either $M_{i}=M_{1}$ or $\mathfrak{P}_{i} \not \mathfrak{P}_{1}$. Put $M^{\prime \prime}=M_{2} \oplus \cdots \oplus M_{n} \oplus \frac{R}{\mathfrak{I}}$. We show that $\left(\mathfrak{P}_{1} M_{1}: \mathfrak{P}_{1} M\right) \nsubseteq \mathfrak{P}_{1}$, and by induction hypothesis $\frac{M}{M_{1}} \cong M^{\prime \prime}$ s.t.r.f., therefore by the previous theorem, $M$ s.t.r.f.

For each $1 \leq i \leq n$, if $M_{i}=M_{1}$, then put $t_{i}=1$. Otherwise consider $r_{i} \in \mathfrak{P}_{i} \backslash \mathfrak{P}_{1}$. Then $r_{i} \in \mathfrak{P}_{i}=\sqrt{\left(0: M_{i}\right)}$, and so there exists $n_{i} \in \mathbb{N}$ such that $r_{i}^{n_{i}} M_{i}=0$. Thus, $t_{i}=$ $r_{i}^{n_{i}} \in\left(\mathfrak{P}_{1} M_{1}: \mathfrak{P}_{1} M_{i}\right) \backslash \mathfrak{P}_{1}$. Let $i \in \mathfrak{I} \backslash \mathfrak{P}_{1}$, and put $r=t_{1} t_{2} t_{3} \cdots t_{n} i$. Then $r \in\left(\mathfrak{P}_{1} M_{1}\right.$ : $\left.\mathfrak{P}_{1} M_{i}\right)$, for each $1 \leq i \leq n$, and whence $r \in\left(\mathfrak{P}_{1} M_{1}: \mathfrak{P}_{1} M\right) \backslash \mathfrak{P}_{1}$, which completes the proof. 
LEMMA 3.9. Suppose that $M^{\prime}=\sum_{i=1}^{t} M_{i}$ is a minimal secondary representation of $M^{\prime}$, where $M_{i}$ is $\mathfrak{P}_{i}$-secondary. Also assume that $\mathfrak{P}_{1}$ is minimal in $\operatorname{Att}(M)$. Let $\mathfrak{I}$ be an ideal of $R$ and $N$ a submodule of $M$. Then $\left(\Im M^{\prime}: N\right) \nsubseteq \mathfrak{P}_{1}$, if and only if $\left(\Im M_{1}: N\right) \nsubseteq \mathfrak{P}_{1}$.

Proof. Assume that there exists an $r \in\left(\mathfrak{I} M^{\prime}: N\right) \backslash \mathfrak{P}_{1}$. If $t=1$, then the proof of the claim is trivial. So assume that $t>1$. By minimality of $\mathfrak{P}_{1}$ and minimality of the representation, no $\mathfrak{P}_{i}$ is a subset of $\mathfrak{P}_{1}$, for $i \neq 1$. Thus, $\mathfrak{P}_{2} \mathfrak{P}_{3} \cdots \mathfrak{P}_{t} \nsubseteq \mathfrak{P}_{1}$. Let $r^{\prime} \in \mathfrak{P}_{2} \ldots \mathfrak{P}_{t} \backslash \mathfrak{P}_{1}$. Then for each $2 \leq i \leq t$, there is a $k_{i} \in \mathbb{N}$ such that $r^{\prime k_{i}} M_{i}=0$. Set $k=\max _{i=2}^{t}\left\{k_{i}\right\}$. Since $r^{\prime} \notin \mathfrak{P}_{1}$, we have ${r^{\prime}}^{k} M^{\prime}=M_{1}$. Now $r^{\prime k} r N \subseteq \mathfrak{I} r^{\prime k} M=\mathfrak{I} M_{1}$ and $r^{\prime k} r \notin \mathfrak{P}_{1}$. The other side of the claim is obvious.

LEMMA 3.10. If $M_{1}$ and $M_{2}$ are submodules of $M$ such that $M_{1} \nsubseteq M_{2}$ and $M_{1}$ is $\mathfrak{P}$-secondary, then $\frac{M_{1}+M_{2}}{M_{2}}$ is $\mathfrak{P}$-secondary.

Proof. The proof is easy and it is left to the reader.

THEOREM 3.11. Assume that $M^{\prime}=\sum_{i=1}^{t} M_{i}$ is a minimal secondary representation of $M^{\prime}$, where $M_{i}$ is $\mathfrak{P}_{i}$-secondary. Also suppose that $\frac{M}{M^{\prime}}$ s.t.r.f. (weakly s.t.r.f.) of degree k. Then $M$ s.t.r.f. (weakly s.t.r.f.) of degree $k$, if one of the following holds:

(a) $k \geq 2$ and $\left(M^{\prime}: \mathfrak{P} M\right) \nsubseteq \mathfrak{P}$, for each maximal element $\mathfrak{P}$ of $\operatorname{Att}(M)$.

(b) $k=1$ and $\left(\mathfrak{P} M^{\prime}: \mathfrak{P} M\right) \nsubseteq \mathfrak{P}$, for each maximal element $\mathfrak{P}$ of $\operatorname{Att}(M)$.

Proof. We state the proof for s.t.r.f., the claim for weakly s.t.r.f. is proved similarly. (a) We use induction on $t$. The base step is true by (3.7)(a). One can easily check that our assumption implies $\left(M^{\prime}: \mathfrak{P} M\right) \nsubseteq \mathfrak{P}$, for every element $\mathfrak{P}$ of $\operatorname{Att}(M)$.

Suppose $\mathfrak{P}_{1}$ is a minimal element of $\operatorname{Att}(M)$. Thus, by applying (3.9) with $\mathfrak{I}=R$ and $N=\mathfrak{P}_{1} M$, we see that $\left(M_{1}: \mathfrak{P}_{1} M\right) \nsubseteq \mathfrak{P}_{1}$. So by (3.7)(a), it suffices to prove $\frac{M}{M_{1}}$ s.t.r.f. of degree $k$.

By (3.10), $\frac{M^{\prime}}{M_{1}}=\sum_{i=2}^{t} \frac{M_{i}+M_{1}}{M_{1}}$ is a secondary representation of $\frac{M^{\prime}}{M_{1}}$. Also, since

$$
\frac{\frac{M}{M_{1}}}{\frac{M^{\prime}}{M_{1}}} \cong \frac{M}{M^{\prime}} \quad \text { and } \quad\left(\frac{M^{\prime}}{M_{1}}: \mathfrak{P} \frac{M}{M_{1}}\right) \supseteq\left(M^{\prime}: \mathfrak{P} M\right) \text {, }
$$

for every $\mathfrak{P} \in \operatorname{Att}(M)$, we can apply the induction hypothesis and get $\frac{M}{M_{1}}$ s.t.r.f. of degree $k$ as required.

(b) Similar to (a), just use (3.7)(b) instead of (3.7)(a) and apply (3.9) with $\mathfrak{I}=\mathfrak{P}_{1}$ instead of $R$.

In [1], it is proved that if $R$ is an integral domain with the Krull dimension one, then every Noetherian representable module s.t.r.f. ([1, Theorem 1.7]). The following corollary generalises this result.

COROLLARY 3.12. Every representable module s.t.r.f.

Proof. Just apply (3.11), with $M^{\prime}=M$, where $M$ is a representable module.

According to [15] an $R$-module $M$ is called special when for each maximal ideal $\mathfrak{M}$ of $R$, each $a \in \mathfrak{M}$ and each $m \in M$, there exist $c \in R \backslash \mathfrak{M}$ and $k \in \mathbb{N}$ such that $c a^{k} m=0$. Semi-simple modules (direct sum of simple modules), locally Artinian modules (modules in which every cyclic submodule is Artinian) and semi-Artinian modules (modules every homomorphic image of which has a non-zero simple submodule) are special (see [15, Section 3]). 
It was proved there that if $M=M^{\prime} \oplus M^{\prime \prime}$, where $M^{\prime}$ is special and $M^{\prime \prime}$ s.t.r.f., then $M$ s.t.r.f. ([15, Theorem 4.8]). Here we will generalise this to s.t.r.f. (weakly s.t.r.f.) of degree $k$. Let $\mathfrak{M}$ be a maximal ideal of $R$ and $k \in \mathbb{N}^{*}$, consider the following property on a submodule $M^{\prime}$ of $M$ :

$\left(\mathrm{III}_{k}\right)$ For every submodule $N$ of $M, R \mathrm{E}_{k}\left(N+\mathfrak{M} M^{\prime}\right) \subseteq \mathcal{F}_{\mathfrak{M}}\left(R \mathrm{E}_{k}(N)\right)$.

LEMMA 3.13. Let $\mathfrak{M}$ be a maximal ideal of $R$ and $M^{\prime}$ a submodule of $M$.

(a) For each submodule $N$ of $M, R E\left(\mathcal{F}_{\mathfrak{M}}(N)\right) \subseteq \mathcal{F}_{\mathfrak{M}}(R E(N))$.

(b) If $M^{\prime}$ satisfies $\left(I I I_{1}\right)$, then it satisfies $\left(I I I_{k}\right)$ for each $0 \neq k \in \mathbb{N}^{*}$.

Proof. (a) It suffices to show that $\mathrm{E}\left(\mathcal{F}_{\mathfrak{M}}(N)\right) \subseteq \mathcal{F}_{\mathfrak{M}}(R \mathrm{E}(N))$, which one can easily prove.

(b) We prove $\left(\mathrm{III}_{k}\right)$ by induction on $k$. Let $N$ be an arbitrary submodule of $M$. For $k>1$, if $N^{\prime}=R \mathrm{E}_{k-1}\left(N+\mathfrak{M} M^{\prime}\right)$, then by induction hypothesis we have $\mathrm{N}^{\prime} \subseteq \mathcal{F}_{\mathfrak{M}}\left(R \mathrm{E}_{k-1}(N)\right)$. Now $R \mathrm{E}_{k}\left(N+\mathfrak{M} M^{\prime}\right)=R \mathrm{E}\left(N^{\prime}\right) \subseteq R \mathrm{E}\left(N^{\prime}+\mathfrak{M} M^{\prime}\right) \subseteq$ $\mathcal{F}_{\mathfrak{M}}\left(R \mathrm{E}\left(N^{\prime}\right)\right) \subseteq \mathcal{F}_{\mathfrak{M}}\left(R \mathrm{E}\left(\mathcal{F}_{\mathfrak{M}}\left(R \mathrm{E}_{k-1}(N)\right)\right)\right)$. Thus, by part (a) of this lemma, $R \mathrm{E}_{k}\left(N+\mathfrak{M} M^{\prime}\right) \subseteq \mathcal{F}_{\mathfrak{M}}\left(\mathcal{F}_{\mathfrak{M}}\left(R \mathrm{E}_{k}(N)\right)\right)=\mathcal{F}_{\mathfrak{M}}\left(R \mathrm{E}_{k}(N)\right)$. The case of $k=\infty$ follows the definition of $R \mathrm{E}_{\infty}(N)$ and the case of $k \in \mathbb{N}$.

Lemma 3.14. Let $k \in \mathbb{N}^{*}$. If $M=M^{\prime} \oplus M^{\prime \prime}$, where $M^{\prime}$ is semi-simple and $M^{\prime \prime}$ s.t.r.f. (weakly s.t.r.f.) of degree $k$, then $M$ s.t.r.f. (weakly s.t.r.f.) of degree $k$.

Proof. In [15], through a series of lemmas, the s.t.r.f. case was proved for $k=1$. In the same way, one can prove the corresponding lemmas for each $k \in \mathbb{N}^{*}$ and weak radicals, and hence prove the claim.

LEMMA 3.15. If $M^{\prime}$ is special, then for every maximal ideal $\mathfrak{M}$ of $R$, (III $)$ holds for $M^{\prime}$.

Proof. This is in fact [15, Lemma 4.7].

THEOREM 3.16. Let $k \in \mathbb{N}^{*}$. If for every maximal ideal $\mathfrak{M}$ of $R$, a submodule $M^{\prime}$ of $M$ satisfies $\left(I I I_{k}\right)$ and $\frac{M}{\mathfrak{M} M^{\prime}}$ s.t.r.f. of degree $k$, then $M$ s.t.r.f. of degree $k$. In particular, if $k \neq 0$ and $M=M^{\prime} \oplus M^{\prime \prime}$, where $M^{\prime}$ is special and $M^{\prime \prime}$ s.t.r.f. of degree $k$, then $M$ s.t.r.f. of degree $k$. The statement also holds if we replace s.t.r.f. with weakly s.t.r.f.

Proof. Let $N$ be an arbitrary submodule of $M, x \in \operatorname{rad}(N)$ and $\mathfrak{I}=\left(R \mathrm{E}_{k}(N): R x\right)$. We must show that $R=\mathfrak{I}$. If $\mathfrak{I} \neq R$, then it is contained in a maximal ideal of $R$, say $\mathfrak{M}$. By our assumption, $\frac{M}{\mathfrak{M} M^{\prime}}$ s.t.r.f. of degree $k$, hence by (3.1), for every maximal ideal $\mathfrak{M}$ of $R, \operatorname{rad}\left(N+\mathfrak{M} M^{\prime}\right) / \mathfrak{M} M^{\prime}=R E_{k}\left(N+\mathfrak{M} M^{\prime}\right) / \mathfrak{M} M^{\prime}$, whence

$$
x \in \operatorname{rad}(N) \subseteq \operatorname{rad}\left(N+\mathfrak{M} M^{\prime}\right)=R \mathrm{E}_{k}\left(N+\mathfrak{M} M^{\prime}\right) \subseteq \mathcal{F}_{\mathfrak{M}}\left(R \mathrm{E}_{k}(N)\right)
$$

This means that there exists an $s \in R \backslash \mathfrak{M}$ such that $s x \in R \mathrm{E}_{k}(N)$. But then $s \in$ $\mathfrak{I} \subseteq \mathfrak{M}$, a contradiction. Thus, $M$ s.t.r.f. of degree $k$.

Now suppose that $k \neq 0$ and $M=M^{\prime} \oplus M^{\prime \prime}$, where $M^{\prime}$ is an special module and $M^{\prime \prime}$ s.t.r.f. of degree $k$. Let $\mathfrak{M}$ be an arbitrary maximal ideal of $R$. According to (3.15) and (3.13), $M^{\prime}$ satisfies $\left(\mathrm{III}_{k}\right)$, for $\mathfrak{M}$. Thus, to complete the proof, we just need to show that $\frac{M}{\mathfrak{M} M^{\prime}}$ s.t.r.f. of degree $k$. But $\frac{M}{\mathfrak{M} M^{\prime}} \cong \frac{M^{\prime}}{\mathfrak{M} M^{\prime}} \oplus M^{\prime \prime}$ and $\frac{M^{\prime}}{\mathfrak{M} M^{\prime}}$ is semi-simple. Thus, the result follows from (3.14). The assertion on satisfying the weak radical formula can be proved similarly.

Next we state a condition on $M^{\prime}$ under which $M$ s.t.r.f. of a higher degree than $\frac{M}{M^{\prime}}$ does. Recall that if $\mathcal{C}: M_{1} \supseteq M_{2} \supseteq \cdots$ is a chain of submodules of $M$, then the 
completion $\widehat{M}$ of $M$ with respect to $\mathcal{C}$ is the set $\left\{\left[\left(x_{i}\right)\right] \mid\left(x_{i}\right)\right.$ is a cauchy sequence in $M$ \}, where $\left[\left(x_{i}\right)\right]$ denotes the equivalency class of the sequence $\left(x_{i}\right)$ with respect to the relation $\left(x_{i}\right) \equiv\left(y_{i}\right)$ if and only if for each $n \in \mathbb{N}$ there is an $n_{0} \in \mathbb{N}$ such that for all $n_{0} \leq i$ we have $x_{i}-y_{i} \in M_{n}$ (see [2, Section 10]). In the particular case that $M_{i}=\mathfrak{I}^{i} M$, for an ideal $\mathfrak{I}$ of $R, \widehat{M}$ is called the $\mathfrak{I}$-adic completion of $M$.

Clearly $\widehat{M}$ forms a module with element-wise operations. In the following, for each submodule $N$ of $M$, we set $\widehat{N}=\left\{\left[\left(x_{i}\right)\right] \in \widehat{M} \mid\left[\left(x_{i}\right)\right]=\left[\left(y_{i}\right)\right]\right.$ for some $y_{i}$ 's in $\left.N\right\}$, which is a submodule of $\widehat{M}$. Moreover, by $\widehat{m}$ for an $m \in M$ we mean $[(m)] \in \widehat{M}$, where $(m)$ denotes a constant sequence, all terms of which equals $m$. Also, we denote the natural homomorphism from $M$ to $\widehat{M}$, which maps $m$ to $\widehat{m}$, by $\widehat{f}$.

THEOREM 3.17. Suppose that $M_{1} \supseteq M_{2} \supseteq \cdots$ is a chain of submodules of $M$ and $\widehat{M}$ is the completion of $M$ with respect to this chain. Also let $N$ be a submodule of $M$.

(a) $\widehat{f}\left(\operatorname{rad}_{M}(N)\right) \subseteq \operatorname{rad}_{\widehat{M}}(\widehat{N})$ and if $M_{l} \subseteq \operatorname{rad}_{M}(N)$, for some $l \in \mathbb{N}$, then $\operatorname{rad}_{\widehat{M}}(\widehat{N}) \subseteq$ $\widehat{\operatorname{rad}_{M}(N)}$

(b) $\widehat{f}\left(E_{M}(N)\right) \subseteq E_{\widehat{M}}(\widehat{N})$ and if $M_{l} \subseteq R E_{k}(N)$, for some $l \in \mathbb{N}$ and $k \in \mathbb{N}^{*}$, then $E_{\widehat{M}}(\widehat{N}) \subseteq R \widehat{E_{k+1}(N)}$

(c) If $M_{l} \subseteq R E_{k}(N)$, for some $l \in \mathbb{N}$ and $k, k^{\prime} \in \mathbb{N}^{*}$ and $\widehat{M}$ s.t.r.f. of degree $k^{\prime}$, then $\frac{M}{N}$ s.t.r.f. of degree $k+k^{\prime}$.

Proof. (a) Let $x \in \operatorname{rad}(N)$. Then by (3.5), for every prime ideal $\mathfrak{P}$ of $R$ there exists an $s \in R \backslash \mathfrak{P}$ such that $s x \in N+\mathfrak{P} M$, say $s x=m+\sum_{i=1}^{t} p_{i} m_{i}$, for some $m_{i}$ 's in $M, p_{i}$ 's in $\mathfrak{P}$ and $m \in N$. Thus, $s \widehat{x}=\widehat{m}+\sum_{i=1}^{t} p_{i} \widehat{m}_{i} \in \widehat{N}+\mathfrak{P} \widehat{M}$. Therefore, according to (3.5) $\widehat{x} \in \operatorname{rad}_{\widehat{M}}(\widehat{N})$.

Now suppose that $M_{l} \subseteq \operatorname{rad}(N)$ and $\left[\left(x_{i}\right)\right] \in \operatorname{rad}_{\widehat{M}}(\widehat{N})$. Then for each prime ideal $\mathfrak{P}$ of $R$ there is an $s \in R \backslash \mathfrak{P}$ such that $s\left[\left(x_{i}\right)\right] \in \widehat{N}+\mathfrak{P} \widehat{M} \subseteq \widehat{N}+\widehat{\mathfrak{P M}}$. So there are $n_{i}$ s in $N$ and $m_{i}$ s in $\mathfrak{P} M$ such that $s\left[\left(x_{i}\right)\right]=\left[\left(n_{i}\right)\right]+\left[\left(m_{i}\right)\right]$. Hence, if $i$ is large enough we have $s x_{i}=n_{i}+m_{i}+\delta_{i}$, where $\delta_{i} \in M_{l} \subseteq \operatorname{rad}(N)$. Consequently, for some $s_{i} \in R \backslash \mathfrak{P}$ we have $s_{i} \delta_{i} \in N+\mathfrak{P} M$. Thus, $s s_{i} x_{i} \in N+\mathfrak{P} M$ and since $\mathfrak{P}$ was arbitrary, we deduce that $x_{i} \in \operatorname{rad}_{M}(N)$ for all large enough is and whence $\left[\left(x_{i}\right)\right] \in \widehat{\operatorname{rad}_{M}(N)}$.

(b) It is obvious that $\widehat{f}\left(\mathrm{E}_{M}(N)\right) \subseteq \mathrm{E}_{\widehat{M}}(\widehat{N})$. So suppose that $M_{l} \subseteq R \mathrm{E}_{k}(N)$ and let $\left[\left(x_{i}\right)\right] \in \mathrm{E}_{\widehat{M}}(\widehat{N})$. Then for some $r \in R,\left[\left(m_{i}\right)\right] \in \widehat{M},\left[\left(n_{i}\right)\right] \in \widehat{N}$ and $t \in \mathbb{N}$, we have $\left[\left(x_{i}\right)\right]=r\left[\left(m_{i}\right)\right]$ and $r^{t}\left[\left(m_{i}\right)\right]=\left[\left(n_{i}\right)\right]$. Therefore, for large enough is we have $r^{t} m_{i}-n_{i} \in$ $M_{l} \subseteq R \mathrm{E}_{k}(N)$. Thus, $r^{t} m_{i} \in R \mathrm{E}_{k}(N)$ and hence $r m_{i} \in R \mathrm{E}_{k+1}(N)$. But for large enough is $x_{i}=r m_{i}+\delta_{i}$, where $\delta_{i} \in M_{l} \subseteq R \mathrm{E}_{k}(N)$. From this we conclude that $x_{i} \in R \mathrm{E}_{k+1}(N)$ for all large enough is, and the result follows.

(c) Let $A$ be a submodule of $M$ containing $N$ and $x \in \operatorname{rad}_{M}(A)$. If $k^{\prime}>0$, then $M_{l} \subseteq R \mathrm{E}_{k}(N) \subseteq R \mathrm{E}_{k}(A) \subseteq \operatorname{rad}(A)$. Therefore, by (a), $\widehat{x} \in \operatorname{rad}_{\widehat{M}}(\widehat{A})=R \mathrm{E}_{k^{\prime}}(\widehat{A})=$

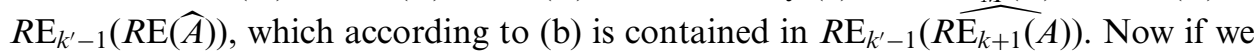
put $B=R \mathrm{E}_{k+1}(A)$, then $M_{l} \subseteq B=R \mathrm{E}_{0}(B)$. So by repeated use of (b) (with 0 as the $k$ in (b)), we get

$$
R \mathrm{E}_{k^{\prime}-1}(\widehat{B})=R \mathrm{E}_{k^{\prime}-2}(R \mathrm{E}(\widehat{B})) \subseteq R \mathrm{E}_{k^{\prime}-2}(\widehat{R \mathrm{E}(B)}) \subseteq \ldots \subseteq R \widehat{\mathrm{E}_{k^{\prime}-1}(B)}=R \widehat{\mathrm{E}_{k^{\prime}+k}(A)}
$$

Therefore, $\widehat{x}=\left[\left(y_{i}\right)\right]$ for some $y_{i} \mathrm{~s}$ in $R \mathrm{E}_{k^{\prime}+k}(A)$, and hence for a large enough $i$, we have $x-y_{i} \in R \mathrm{E}_{k}(N)$. Consequently, $x \in R \mathrm{E}_{k^{\prime}+k}(A)$, as required. If $k^{\prime}=0$, then $\widehat{x} \in \widehat{A}$ and by an argument similar to that of (b), we get $x \in R \mathrm{E}_{k}(A)$. 
COROLlARY 3.18. Let $\mathfrak{I}$ be a finitely generated ideal of $R$ and $k, k^{\prime} \in \mathbb{N}^{*}$. If $\mathfrak{I} \subseteq$ $\sqrt{\left(R E_{k}(0): M\right)}$ and the $\mathfrak{I}$-adic completion of $M$ (as an R-module) s.t.r.f. of degree $k^{\prime}$, then $M$ s.t.r.f. of degree $k+k^{\prime}$.

Proof. Note that since $\mathfrak{I}$ is finitely generated, for some $n \in \mathbb{N}$ we have $\mathfrak{I}^{n} \subseteq\left(R E_{k}(0)\right.$ : $M)$. Hence, the result follows from (3.17).

PROPOSITION 3.19. If $M^{\prime} \subseteq R \mathrm{E}_{k}(0)$ and $\frac{M}{M^{\prime}}$ s.t.r.f. (weakly s.t.r.f.) of degree $k^{\prime}$, where $k, k^{\prime} \in \mathbb{N}^{*}$, then $M$ s.t.r.f. (weakly s.t.r.f.) of degree $k+k^{\prime}$.

Proof. Noting that for every submodule $N$ of $M$, we have $M^{\prime} \subseteq R \mathrm{E}_{k}(0) \subseteq$ $R \mathrm{E}_{k}(N) \subseteq \operatorname{wrad}(N)=\operatorname{wrad}\left(R \mathrm{E}_{k}(N)\right)$, the result for weak radicals follows (3.1). The case on radicals can be proved similarly or can be deduced from (3.17), setting $M_{i}=M^{\prime}$ for all $i \in \mathbb{N}$.

COROLlary 3.20. Let $R$ be a one-dimensional ring.

(a) If $R$ is a domain and $\left(R E_{k}(0): m\right) \neq 0$ for each $m \in M$, then $M$ s.t.r.f. of degree $k+1$. Moreover, if $\left(R E_{k}(0): M\right)$ is a non-zero radical ideal, then $M$ s.t.r.f. of degree $k$.

(b) If $\left(R E_{k}(0): M\right)$ is a prime ideal, then $M$ weakly s.t.r.f. of degree $k+1$.

Proof. (a) By our hypothesis, $\frac{M}{R \mathrm{E}_{k}(0)}$ is a torsion module. By [15, Corollary 3.6], every torsion module over a one-dimensional domain is special and hence s.t.r.f. Now use (3.19). For the second part, note that by [16, Theorem 2.4] if $\left(R \mathrm{E}_{k}(0): M\right)$ is a non-zero radical ideal, then $\frac{M}{R \mathrm{E}_{k}(0)}$ s.t.r.f. of degree 0 .

(b) This follows from (3.19) and [5, Corollary 3.3], which states that every onedimensional domain weakly s.t.r.f.

The following corollary is proved in [14] but we bring it here for completeness.

COROLlary 3.21. Assume that $R$ is a Noetherian ring. The following are equivalent.

(a) $R$ has the s.p.a.r. property.

(b) R s.t.r.f. of degree $k$, for some $2 \leq k \in \mathbb{N}^{*}$.

(c) R s.t.r.f. of degree 2.

(d) $R$ is an Artinian ring, or all of the following conditions are satisfied, where $\mathfrak{P}_{1}, \ldots, \mathfrak{P}_{n}$ are all of the minimal ideals of $R$ :

(1) $\operatorname{dim} R=1$ and for each $i, \frac{R}{\mathfrak{P}_{i}}$ is a Dedekind domain.

(2) If $n \geq 2$, then for each $k=1,2, \cdots, n-1,\left(\bigcap_{i=1}^{k} \mathfrak{P}_{i}\right)+\mathfrak{P}_{k+1}=\bigcap_{i=1}^{k}\left(\mathfrak{P}_{i}+\right.$ $\left.\mathfrak{P}_{k+1}\right)$.

(3) If $n \geq 2$, then for each $i, j, 1 \leq i<j \leq n, R=\mathfrak{P}_{i}+\mathfrak{P}_{j}$, or $\frac{R}{\mathfrak{P}_{i}+\mathfrak{P}_{j}}$ is a semisimple Artinian ring.

Proof. As it was noted in the Introduction, $R$ has the s.p.a.r. property if and only if $R$ s.t.r.f. of degree $\infty$. So (b) $\Rightarrow$ (a) and also (c) $\Rightarrow($ b), are clear. Thus, we just need to show that if $M$ has the s.p.a.r. property, then $M$ s.t.r.f. of degree 2. Suppose that $R$ has the s.p.a.r. property and $M$ is an arbitrary $R$-module. Now $\mathrm{N}(R) M \subseteq R \mathrm{E}(0)$, where $\mathrm{N}(R)$ stands for the nil radical of $R$, and hence $\frac{M}{R \mathrm{E}(0)}$ is an $\frac{R}{\mathrm{~N}(R)}$-module. Since every $\frac{R}{\mathrm{~N}(R)}$-module is an $R$-module, $\frac{R}{\mathrm{~N}(R)}$ has the s.p.a.r. property and hence by (2.4), s.t.r.f. Thus, $\frac{M}{R \mathrm{E}(0)}$ s.t.r.f. and by (3.19) $M$ s.t.r.f. of degree 2 .

(a) $\Leftrightarrow(d)$ The proof is given by [10, Theorem 1.1]. 
EXAMPLE 3.22. In [10, p. 33] it was noted that $R=\frac{F[x, y]}{<x^{2} y^{2}>}$ has the s.p.a.r. property but does not s.t.r.f. So by the previous corollary $R$ s.t.r.f. of degree 2 but does not s.t.r.f. of degree 1 .

At the end of this paper we turn our attention to a stronger form of radical formula. In [6] we stated that $M$ satisfies the simplified radical formula (s.t.s.r.f.) if $\operatorname{rad}_{M}(N)=\mathrm{E}_{M}(N)+N$, for each submodule $N$ of $M$. If every $R$-module s.t.s.r.f., then we say that $R$ s.t.s.r.f. In [6, Theorem 2.15], it is proved that a Noetherian ring s.t.s.r.f. if and only if it is a ZPI-ring. Here we consider the generalisation of the above concept.

Definition 3.23. Let $k \in \mathbb{N} \cup\{0\}$. If $\operatorname{rad}_{M}(N)=k \mathrm{E}_{M}(N)+N\left(\operatorname{wrad}_{M}(N)=\right.$ $\left.k \mathrm{E}_{M}(N)+N\right)$ for every submodule $N$ of $M$ then we say that $M$ satisfies (weakly satisfies) the simplified radical formula of degree $k$ (s.t.s.r.f. (weakly s.t.s.r.f.) of degree $k$ ). Also we say that $R$ satisfies (weakly satisfies) the simplified radical formula of degree $k$, when every $R$ module does so.

Recall that $\mathrm{E}_{M}(N)$ is not necessarily a submodule of $M$, and $k \mathrm{E}_{M}(N)$ in the above definition is

$$
k \mathrm{E}_{M}(N)=\left\{x \in M \mid \exists x_{1}, x_{2}, \cdots, x_{k} \in E_{M}(N) \ni x=x_{1}+x_{2}+\cdots+x_{k}\right\} .
$$

In [5] we have proved that a valuation domain of the Krull dimension $k$, s.t.s.r.f. of degree $k$ but not of $k-1$ and also we have shown that if $R$ s.t.s.r.f. of degree $k$, then $\operatorname{dim} R \leq k$.

LEMMA 3.24.

(a) Let $K \subseteq N$ be submodules of $M$. Then $\operatorname{rad}_{\frac{M}{K}}\left(\frac{N}{K}\right)=k E_{\frac{M}{K}}\left(\frac{N}{K}\right)+\frac{N}{K}$ if and only if $\operatorname{rad}_{M}(N)=k E_{M}(N)+N$.

(b) Let $k \in \mathbb{N}^{*}$. If $M=M^{\prime} \oplus M^{\prime \prime}$, where $M^{\prime}$ is semi-simple and $M^{\prime \prime}$ s.t.s.r.f. (weakly s.t.s.r.f.) of degree $k$, then $M$ s.t.s.r.f. (weakly s.t.s.r.f.) of degree $k$.

Proof. (a) is easy and (b) can be proved similar to [15, Lemma 4.5], using (a) instead of [15, lemma 4.1].

A ring $R$ is called weakly Bezout when every finitely generated proper ideal of $R$ is contained in a proper principal ideal (see [6]).

THEOREM 3.25. Let $(R, \mathfrak{M})$ be a local weakly Bezout ring, $k \in \mathbb{N} \cup\{0\}$ and $M=$ $M^{\prime} \oplus M^{\prime \prime}$, where $M^{\prime}$ and $M^{\prime \prime}$ are submodules of $M$. If $M^{\prime}$ is a special module and $M^{\prime \prime}$ s.t.s.r.f. (weakly s.t.s.r.f.) of degree $k$, then $M$ s.t.s.r.f. (weakly s.t.s.r.f.) of degree $k+1$.

Proof. First suppose that $x \in \mathfrak{M} M^{\prime}$, say $x=\sum_{i=1}^{t} r_{i} m_{i}$ for some $r_{i}$ in $\mathfrak{M}$ and some $m_{i}$ s in $M^{\prime}$. Then $\mathfrak{I}=<r_{1}, r_{2}, \ldots, r_{n}>\subseteq \mathfrak{M}$, and since $R$ is weakly Bezout, $\mathfrak{I} \subseteq R r$ for some $r \in \mathfrak{M}$, say $r_{i}=a_{i} r$ for some $a_{i}$ s in $R$. Then $x=r \sum_{i=1}^{t} a_{i} m_{i}$ and because $M^{\prime}$ is special and $r \in \mathfrak{M}$, there is a $t^{\prime} \in \mathbb{N}$ such that $r^{t^{\prime}} \sum_{i=1}^{t} a_{i} m_{i}=0$. Therefore, $\mathfrak{M} M^{\prime} \subseteq$ $\mathrm{E}_{M}(0)$.

Since $M=M^{\prime} \oplus M^{\prime \prime}, \frac{M}{\mathfrak{M} M^{\prime}} \cong \frac{M^{\prime}}{\mathfrak{M} M^{\prime}} \oplus M^{\prime \prime}$. As $\frac{M^{\prime}}{\mathfrak{M} M^{\prime}}$ is semi-simple, by (3.24)(b) $\frac{M}{\mathfrak{M} M^{\prime}}$ s.t.s.r.f. of degree $k$. Consequently if $N$ is a submodule of $M$, then $\operatorname{rad}(N) \subseteq$ $\operatorname{rad}\left(N+\mathfrak{M} M^{\prime}\right)=k \mathrm{E}\left(N+\mathfrak{M} M^{\prime}\right)+N+\mathfrak{M} M^{\prime}$. We will prove that $\mathrm{E}\left(N+\mathfrak{M} M^{\prime}\right) \subseteq$ $\mathrm{E}(N)+\mathfrak{M} M^{\prime}$. Then $\operatorname{rad}(N) \subseteq k \mathrm{E}(N)+N+\mathfrak{M} M^{\prime} \subseteq(k+1) \mathrm{E}(N)+N$ and the result follows. 
Let $x \in \mathrm{E}\left(N+\mathfrak{M} M^{\prime}\right)$. Then $x=r m$ and $r^{t} m=n+r^{\prime} m^{\prime}$ for some $r \in R, \quad r^{\prime} \in$ $\mathfrak{M}, n \in N, m \in M, m^{\prime} \in M^{\prime}$ and $t \in \mathbb{N}$. If $r$ is a unit, then $x \in N+\mathfrak{M} M^{\prime}$ as required. Suppose that $r \in \mathfrak{M}$. Note that $M^{\prime}$ is a special module, then there exists $t^{\prime} \in \mathbb{N}$ with $r^{t^{\prime}} m^{\prime}=0$. Now $r^{t+t^{\prime}} m=r^{t^{\prime}} n+r^{\prime} r^{t^{\prime}} m^{\prime} \in N$, whence $x=r m \in \mathrm{E}(N)$, which completes the assertion. The case for weak radicals is quite similar.

ACKNOWLEDGEMENTS. The authors would like to thank the referee for his valuable comments and suggestions.

\section{REFERENCES}

1. M. Alkan and Y. Tiraş, On prime submodules, Rocky Mount. J. Math. 37(3) (2007), $709-722$

2. M. F. Atiyah and I. G. Macdonald, Introduction to commutative algebra (AddisonWesley, Boston, MA, 1969).

3. A. Azizi, Radical formula and prime submodules, J. Algebra 307 (2007), 454-460. 405-412.

4. A. Azizi, Radical formula and weakly prime submodules, Glasgow Math. J. 51 (2009),

5. A. Azizi and A. Nikseresht, Prime bases of weakly prime submodules and the radical formula, Comm. Algebra, submitted for publication, $29 \mathrm{pp}$.

6. A. Azizi and A. Nikseresht, Simplified radical formula in modules, Houston J. Math., to appear, $12 \mathrm{pp}$. 195.

7. M. Behboodi and H. Koohi, Weakly prime modules, Vietnam J. Math. 32 (2004), 185-

8. M. D. Larsen and P. J. McCarthy, Multiplicative theory of ideals (Academic Press, Oxford, UK, 1971).

9. K. H. Leung and S. H. Man, On commutative Noetherian rings which satisfy the radical formula, Glasgow Math. J. 39 (1997), 285-293.

10. S. H. Man, On commutative Noetherian rings which have the s.p.a.r. property, Arch. Math. J. 70 (1998), 31-40.

11. S. H. Man, On commutative Noetherian rings which satisfy the generalized radical formula, Comm. Algebra 27(8) (1999), 4075-4088. 1992).

12. H. Matsumura, Commutative ring theory (Cambridge University Press, Cambridge, UK,

13. R. McCasland and M. Moore, On radicals of submodules of finitely generated modules, Canad. Math. Bull. 29(1) (1986), 37-39.

14. A. Parkash, Prime submodules and radical formulae, Contrib. Algebra. Geom. (Beiträge Algebra Geom.), to appear, $8 \mathrm{pp}$.

15. D. Pusat-Yilmaz and P. F. Smith, Modules which satisfy the radical formula, Acta. Math. Hungar. 95 (2002), 155-167.

16. H. Sharif, Y. Sharifi and S. Namazi, Rings satisfying the radical formula, Acta Math. Hungar. 71 (1996), 103-108. 